DOI 10. 18307/2017. 0625

(c) 2017 by Journal of Lake Sciences

\title{
大别山库区降水预报性能评估及应用对策
}

\author{
叶金印 ${ }^{1,2}$, 张锦堂 $^{3}$, 黄 勇 $^{2}$, 安晶晶 ${ }^{1}$, 叶正阳 $^{4}$ \\ (1: 安徽省气象台, 合肥 230031) \\ (2: 安徽省大气科学与卫星遥感重点实验室, 合肥 230031) \\ (3:安徽省水文局,合肥 230031) \\ (4:南京信息工程大学大气科学学院, 南京 210044)
}

摘 要: 对降水预报进行性能评估及应用对策研究可以更好地发挥降水预报在水库调度中的决策支持作用. 基于大别山 库区近 $10 \mathrm{a}$ 汛期(2007-2016 年 5月 1 日-9 月 30 日) 24 168 h 共 7 个预见期降水预报和地面降水观测资料, 采用正确 率、TS 评分、概率统计、ROC 曲线以及 $C T S$ 等方法评估大别山库区降水预报性能, 并以响洪甸水库为重点研究区域分析降 水预报在水库调度中的应用对策. 结果表明: 1) 大别山库区各量级的降水预报都有正预报技巧;24 72 h 预见期降水预报 的 TS 评分较高且空报率、漏报率也较低,具有较高的预报性能; 但 $96 \mathrm{~h}$ 及以上预见期降水预报性能明显下降,中雨以上 量级空报率、漏报率较大, 特别是对大暴雨及其以上量级的降水预报性能显著下降. 2) 大别山库区预报降水量级与实况 降水量级基本符合, 预报降水量级大于等于实况降水量级的概率超过 $75 \%$; 虽然降水预报量级上呈现出过度预报的现 象, 但降水过程预报对水库调度仍有较好的应用价值, 应用时要考虑到降水预报量级可能存在偏差. 3) 转折性天气预报 $96 \mathrm{~h}$ 及以上预见期 CTS 评分较低,但 $72 \mathrm{~h}$ 以内预见期的性能明显改进,尤其是 $24 \mathrm{~h}$ 预见期 CTS 评分也提高到了 $38.2 \%$; 水库调度可从长预见期的降水预报获取降水过程及其可能发生转折的信息, 根据短预见期的降水预报进行调度方案 调整.

关键词: 大别山库区; 降水预报; 性能评估; 水库调度;应用对策; 响洪甸水库

\section{Evaluation of precipitation forecasts for Dabie Mountain area and application counter- measures for the reservoirs regulation}

\author{
YE Jinyin ${ }^{1,2}$, ZHANG Jintang ${ }^{3}$, HUANG Yong ${ }^{2}$, AN Jingjing ${ }^{1} \&$ YE Zhengyang $^{4}$ \\ (1: Anhui Meteorological Observatory, Hefei 230031, P.R.China) \\ (2: Anhui Key Lab of Atmospheric Science and Satellite Remote Sensing, Hefei 230031, P.R.China) \\ (3: Anhui Hydrology Bureau, Hefei 230031, P.R.China) \\ (4: College of Atmospheric Science, Nanjing University of Information Science and Technology, Nanjing 210044, P.R.China)
}

Abstract: The evaluation and application research of precipitation forecasts for reservoirs area can help to optimize the reservoir regulation. Based on the precipitation forecasts with 7 lead time $(24-168 \mathrm{~h})$ and the observation precipitation data in the flood season during 2007-2016, evaluation methods (e.g. Percentage Correct, Threat Score, Probability Statistics, ROC curve, CTS) were introduced to estimate the precipitation prediction skill, and the application countermeasures for the reservoir regulation in Xianghongdian Reservoir of Dabie Mountain area were analyzed. The results indicate that: 1) The precipitation forecasting skills for all precipitation magnitudes are all positive in the Dabie Mountains reservoir area. The precipitation forecast performance of 24-72 $\mathrm{h}$ lead time in the Dabie Mountains reservoir area is the best, and the TS score is relatively high and false alarm rate and missing forecast rate are also relatively low. However, the forecasting performance of $96 \mathrm{~h}$ lead time and above is obviously decreased, the false alarm rate and missing forecast rate are getting higher for moderate rain, especially for heavy rain. 2) Overall, even though the rainfall scale predictions are generally consistent with the observations, the probability of precipitation forecast greater than or equal to the observation is more than $75 \%$. Although it is inclined to the over forecast the precipitation scale, the precipitation forecast

* 公益性行业(气象)科研专项(GYHY201406021) 资助. 2017-01-17 收稿;2017-03-16 收修改稿. 叶金印( 1968 ), 男,正研级高级工程师,博士;E-mail: yejinyin@ sina.com. 
products still havea good application value. However, precipitation scale forecast deviation must be considered during application. 3 ) Compared to the forecasts with long lead time which have great uncertainty, forecasts with short lead time are more reliable and have better performance in heavy precipitation processes and transition weather. The CTS scores of $96 \mathrm{~h}$ lead time and above are relatively low , but the forecasting performance is improved significantly within $72 \mathrm{~h}$ lead time. Especially, the CTS scores of $24 \mathrm{~h}$ lead time increases to $38.2 \%$. In practical application, the combination of precipitation forecasts with different forecast periods, including the latest forecasts, are strongly suggested to get the information of transition weather. Such a forecast combination can be utilized to adjust the reservoirs regulation timely when the transition weather is approaching. Our statistic results have justified the usability of precipitation forecast, and the research results could be a valuable reference for the reservoir regulation decision making. Keywords: Dabie Mountain area; precipitation forecast; performance evaluation; reservoir regulation; application countermeasures; Xianghongdian Reservoir

降水预报是防汛抗旱决策的重要科学依据 ${ }^{[-2]}$, 利用天气预报进行科学调度和防控是防汛抗旱重要的 非工程措施之一 ${ }^{[3-4]}$. 水库作为防汛抗旱主要工程性措施,通过削峰填谷,在时空上重新分配水量,达到防洪 错峰、蓄水兴利的目的,实现洪水资源安全利用 ${ }^{[5]}$. 选用不同预见期降水预报信息,采用工程性措施与非工 程性措施相结合的方法可为洪水资源利用、防汛抗洪物资调配提前提供决策支持信息, 为抗洪抢险赢得宝 贵时间 ${ }^{[6]}$.

根据降水预报信息可提高人库洪量、洪水人库流量过程预报精度和延长预见期, 以提前对洪水过程进 行调度分析, 确定是否进行预泄洪水或蓄水, 以最大限度地减少洪水风险和实现洪水资源利用 ${ }^{[7-8]}$. 准确地 把握降水预报性能并有效利用降水预报, 对于水库科学调度, 保障人民生命财产安全和发挥经济社会效益 有重要价值 ${ }^{[9-11]}$.

大别山区属于典型的亚热带季风气候区, 具有气候温和、雨量充沛的特点. 尤其汛期北方大陆性气团和 南方暖湿气团在此交汇, 亦常常受到内陆台风低压环流的影响, 而且山区地形抬升对降雨量的增幅较明显, 在大别山区形成一个多雨中心, 造成该地区山洪灾害频发 ${ }^{[12]}$, 尤其是大别山北麓的淠、史河上游洪水亦会造 成淮河流域中下游地区大范围洪水, 严重影响和制约了淮河流域社会经济可持续发展 ${ }^{[13-14]}$. 因此,把握大别 山库区降水预报性能并将其运用到水库调度, 对于减轻大别山区山洪灾害及发挥水库防洪、蓄水灌溉和发 电效益有着重要的现实意义 ${ }^{[15-16]}$.

本文对近 $10 \mathrm{a}$ 大别山库区降水预报进行性能评估, 并以响洪甸水库为重点研究区域分析水库调度应用 对策, 以更好地发挥降水预报在水库防洪、蓄水灌溉发电调度工作中的决策支持作用.

\section{1 研究区域与资料}

以大别山区北麓娚河、史河上游的梅山、响洪甸、佛子岭、磨子潭和白莲崖等 5 座水库集水区为降水预 报性能评估研究区域. 水库集水区面积为 $5210 \mathrm{~km}^{2}$, 其中梅山水库位于史河上游, 响洪甸水库位于㴓河西源 支流上游, 佛子岭水库位于㴓河东源支流, 磨子潭、白莲崖为佛子岭水库上游的梯级水库 (图 1). 以响洪甸 水库为重点研究区域,分析降水预报在水库调度中的应用对策.

选择霍山、金寨 2 个国家级气象站作为研究大别山库区降水预报性能的代表站, 2007-2016 年 5 月 1 日-9 月 30 日的逐日降水分级预报资料来源于安徽省气象台，逐日降水预报划分为 7 个预见期: $0 \sim 24 、 24 \sim$ $48 、 48 \sim 72 、 72 \sim 96 、 96 \sim 120 、 120 \sim 144 、 144 \sim 168 \mathrm{~h}$ (北京时间 20 时为日界, 下同). 2 个国家级气象站地面降 水观测资料采用安徽省气象信息中心提供的整编资料.

\section{2 降水预报性能评估方法}

采用中国气象局《中短期天气预报质量检验办法》中规定的正确率 (Percentage Correct, $P C$ )、 $T S$ 评分 (Threat Score, 风险评分, 简称 $T S$ )、漏报率 $(P O)$ 、空报率 $(F A R)$ 等业务评分方法, 以及 $R O C$ 曲线 (Receiver Operating Characteristic Curve, 相对特征运行曲线)、概率统计、转折天气评分 (CTS) 等方法评估无雨、小雨、 中雨、大雨、暴雨、大暴雨、特大暴雨 7 个量级的降水预报. 降水量分级采用中国气象局日降水量等级划分标 准: 小雨 $(0.1 \sim 9.9 \mathrm{~mm})$ 、中雨 $(10.0 \sim 24.9 \mathrm{~mm})$ 、大雨 $(25.0 \sim 49.9 \mathrm{~mm})$ 、暴雨 $(50.0 \sim 99.9 \mathrm{~mm})$ 、大暴雨 $(100.0 \sim$ 


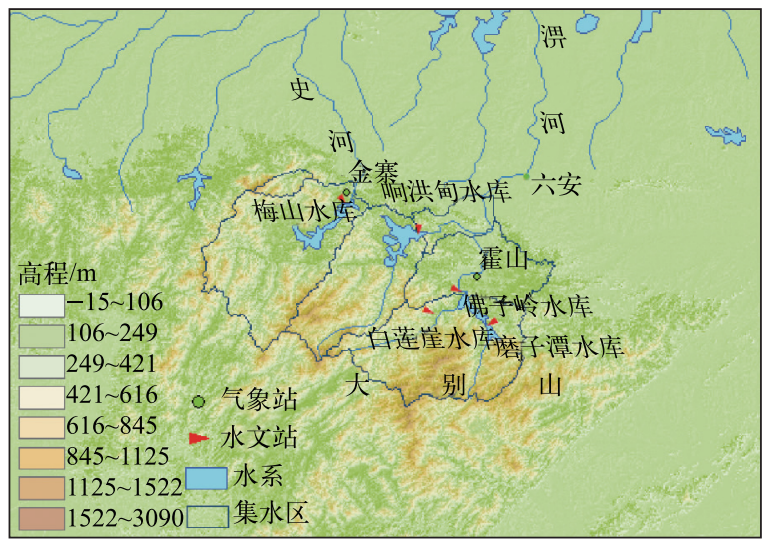

图 1 大别山水库集水区及气象水文站点

Fig.1 The reservoir watersheds in Dabie Mountain area and the locations of rain gauge and streamflow stations

表 1 预报与实况列联表 *

Tab. 1 The contingency table of the dimorphic distribution

\begin{tabular}{ccc}
\hline & 预报 (有) & 预报 (无) \\
\hline 实况(有) & $N A$ & $N C$ \\
实况(无) & $N B$ & $N D$ \\
\hline
\end{tabular}

* $N A$ 为预报正确次数, $N B$ 为空报次数, $N C$ 为漏 报次数, $N D$ 为 “无降水” 预报正确次数.
$250.0 \mathrm{~mm}$ ) 和特大暴雨 $(>250 \mathrm{~mm})$.

对 2 个国家级气象站逐日预报中“有” “无” (某量级) 降 水和实况 “有” “无” (某量级) 降水结果构成预报与实况列联 表(表 1).

\section{1 正确率}

“有” “无” 降水预报 (晴雨预报) 正确率 $(P C)$ 计算公 式为:

$$
P C=(N A+N D) /(N A+N B+N C+N D) \times 100 \%
$$

式中, $N A 、 N B 、 N C 、 N D$ 含义见表 1 , 下同.

\section{$2.2 T S$ 评分、漏报率以及空报率}

$T S$ 评分 $(T S)$ 、漏报率 $(P O)$ 以及空报率 $(F A R)$ 计算公式分别为:

$$
\begin{gathered}
T S_{k}=N A_{k} /\left(N A_{k}+N B_{k}+N C_{k}\right) \times 100 \% \\
P O_{k}=N C_{k} /\left(N A_{k}+N C_{k}\right) \times 100 \% \\
F A R_{k}=N B_{k} /\left(N A_{k}+N B_{k}\right) \times 100 \%
\end{gathered}
$$

式中, $k$ 代表降水量级. 漏报率为实况量级高于预报量级在预报中的比例, 说明了预报不足的现象. 空报率为 实况量级低于预报量级在预报中的比例, 反映了预报过度的现象. TS 评分为实况与预报量级一致在预报中 的比例,数值越大表明预报越准确.

\section{3 概率统计}

各等级降水预报对应的实际降水量级概率 $(P)$ 计算公式为：

$$
P_{k}=N_{k i} / N_{k} \times 100 \%
$$

式中, $N_{k}$ 为预报 $k$ 等级降水次数, $N_{k i}$ 为预报 $k$ 等级降水预报时实际发生的 $i$ 等级降水次数.

\section{$2.4 R O C$ 曲线}

相对特征运行曲线 ( $R O C$ 曲线) 分析方法广泛应用于概率天气预报技巧检验 ${ }^{[17]} . R O C$ 曲线是以虚警率 为横坐标,命中率为纵坐标绘制的曲线. 命中率 $\left(R_{\mathrm{hit}}\right)$ 、虚警率 $\left(R_{\mathrm{falsealarm}}\right)$ 计算公式分别为:

$$
\begin{gathered}
R_{\text {hit }}=N A /(N A+N C) \times 100 \% \\
R_{\text {falsealarm }}=N B /(N B+N D) \times 100 \%
\end{gathered}
$$

$R O C$ 曲线和横坐标构成的面积为 $A U C$ 值 (Area Under Curve), $R O C$ 曲线位于对角线上方时, 命中率大于 虚警率, 即当 $A U C>0.5$ 时,有正的预报价值.

\section{5 转折性天气评分}

转折性天气是指从无到有或者从有到无的转折性降水过程. 若预报与实况一致, 则判定转折性天气预报 
正确; 预报出现转折天气而实况没有出现则判定为空报, 实况出现了转折性天气而未能预报则判定为漏报 ${ }^{[18]}$. 转折性天气预报评分 $(C T S)$ :

$$
C T S=C N A /(C N S+C N Y-C N A) \times 100 \%
$$

转折天气空报率:

$$
C F A R=(C N Y-C N A) / C N Y \times 100 \%
$$

转折天气漏报率:

$$
\text { CPO }=(C N S-C N A) / C N S \times 100 \%
$$

式中, $C N S$ 为实况转折性天气的数量, $C N Y$ 为预报转折性天气的数量, $C N A$ 为实况和预报均为转折性天气, 并且转折过程类型相同.

\section{3 降水预报评估指标分析}

对 2007-2016 年汛期 (5 月 1 日-9 月 30 日) 逐年实况降水观测数据以及 $24 \sim 168 \mathrm{~h}$ 预见期降水 预报数据分不同量级和预见期进行统计. 因特大暴 雨出现的次数非常少, $10 \mathrm{a}$ 来也未对大别山库区未 作出过 “特大暴雨” 预报, 为便于分析将 “特大暴雨” 合并到 “大暴雨” 进行处理分析. 对 7 个预见期内 “有” “无” 降水预报 (不分量级) 统计晴雨预报正确 率, 对各降水量级不分预见期计算 $T S$ 评分、空报率 和漏报率(图 2).

晴雨预报正确率为 $74.3 \%$, 表明在不考虑量级 的情况下, 晴雨预报的性能较高. 小雨、中雨、大雨、 暴雨、大暴雨量级对应的 TS 评分分别为: $39.0 \%$ 、 $13.2 \% 、 9.4 \% 、 9.0 \%$ 和 $2.0 \%$, 随着降水量级增大明显

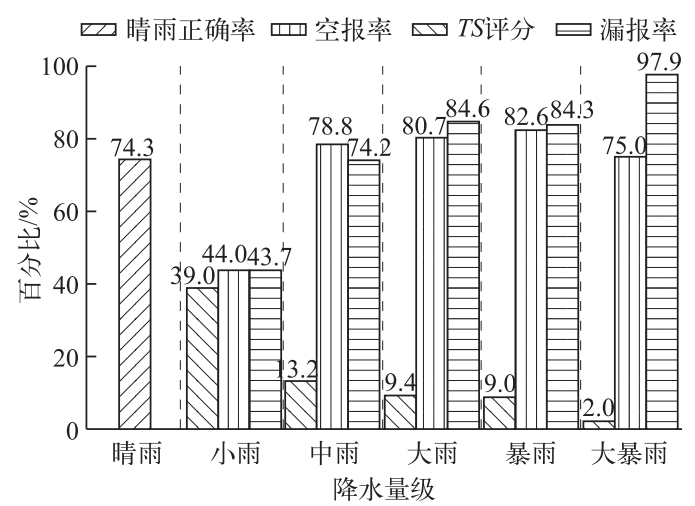

图 2 不同降水量级预报的 TS 评分、空报率和漏报率

Fig.2 TS score, false alarm rate and missing forecast rate of different level precipitation forecasts

下降, 空报率和漏报率明显提升. 大别山库区汛期以锋面和江淮气旋降雨为最多, 由于此类降水系统雨区范 围大, 降雨量空间变异性相对较小, 预报性能相对较高; 切变线、低空急流、低浴及台风是产生大别山库区强 降水的主要天气系统, 此类天气系统的强雨带和雨量中心位置不确定性大; 此外, 大别山地形地貌不仅对降

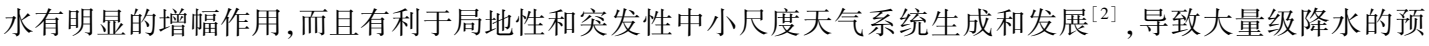
报难度较高, 对应的 $T S$ 评分随之降低.

大别山库区降水预报性能随预见期延长呈现整体下降趋势 (图 3). 24 72 h 预见期, TS 评分较高且空 报率、漏报率也较低, 具有较高的预报性能; $96 \mathrm{~h}$ 及以上预见期, 中雨以上量级空报率、漏报率较大, 降水预 报性能明显下降, 特别是对大暴雨及其以上量级的降水预报性能显著下降. 各预见期晴雨预报正确率均在 $64 \%$ 以上,表明对降水范围的预报能力较高, 但对强降水中心的预报能力较差.

\section{4 降水预报在水库调度中的应用对策分析}

降水预报具有较大的误差和不确定性, 主要体现在位置偏差、量级偏差以及降水开始或结束时间的偏 差,并且这种偏差随时间累积而增大. 深人认知降水预报的性能,发掘降水预报的有效性和确定性信息, 可 为水库调度提供科学的决策支持信息.

\section{1 降水分级预报应用策略}

选择所有预见期预报有降水以及实际发生降水的天气个例构成统计样本, 分析各量级降水预报对应的 实际降水量级概率以及各量级预报下的降水量期望值 (表 2). 预报降水量级主要分布在实况降水量级附近 及以上, 预报降水量级大于等于实况降水量级的概率超过 $75 \%$, 预报量级上呈现出过度预报的特点. 随预报 量级的增大, 实况出现小雨或无雨的概率明显减小, 而实况出现中雨、大雨、暴雨、大暴雨的概率明显上升, 说明对大别山库区的降水过程预报具有较好的性能. 

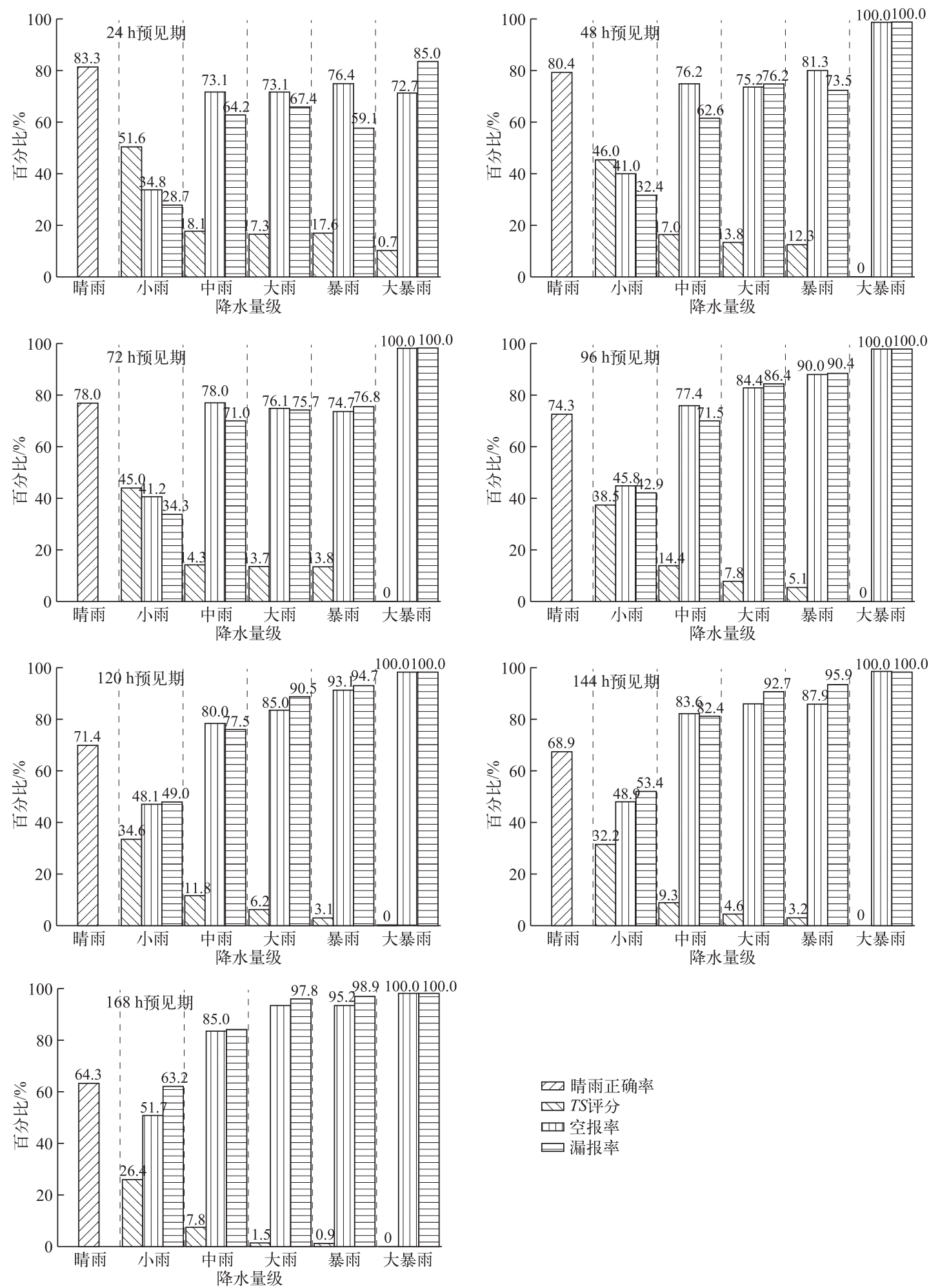

图 3 不同预见期各量级降水预报 TS 评分、空报率和漏报率

Fig.3 TS score, false alarm rate and missing alarm rate of different level precipitation forecasts in different lead time 
表 2 各降水量级预报对应实际发生的降水量级概率及降水量期望值

Tab.2 The actual precipitation level probability in each precipitation level forecast and rainfall expectation

\begin{tabular}{|c|c|c|c|c|c|c|c|}
\hline \multirow{2}{*}{ 预报等级 } & \multicolumn{5}{|c|}{ 降水概率 } & \multirow{2}{*}{$\begin{array}{c}\text { 最小期望值/ } \\
\mathrm{mm}\end{array}$} & \multirow{2}{*}{$\begin{array}{c}\text { 最大期望值 } \\
\mathrm{mm}\end{array}$} \\
\hline & 小雨/\% & 中雨 $/ \%$ & 大雨/\% & 暴雨/\% & 大暴雨/\% & & \\
\hline 小雨 & 41.0 & 13.3 & 8.1 & 4.0 & 1.0 & 6.77 & 17.91 \\
\hline 中雨 & 40.6 & 18.8 & 13.9 & 7.8 & 2.2 & 11.86 & 28.93 \\
\hline 大雨 & 36.5 & 19.9 & 17.4 & 11.8 & 3.0 & 15.61 & 36.54 \\
\hline 暴雨 & 32.3 & 19.7 & 19.2 & 15.4 & 5.6 & 20.39 & 47.07 \\
\hline 大暴雨 & 19.5 & 15.0 & 22.5 & 31.5 & 9.5 & 32.57 & 72.11 \\
\hline
\end{tabular}

对于水库调度而言, 需根据不同量级的降水 预报作出不同的响应. 以大别山库区响洪甸水库 2016 年 6 月 12 日 2 时水位 ( $125 \mathrm{~m}$, 汛限水位) 为 起点, 此时前期影响降雨量为 $18.9 \mathrm{~mm}$, 以未来 24 $\mathrm{h}$ 降水预报 (取各量级雨量上限) 为驱动, 采用水 利部淮河水利委员会编的《淮河流域淮河水系实 用水文预报方案》中的降雨径流关系模型 ${ }^{[19]}$ 计算 相同降雨径流关系下的人库流量过程线 (图 4). 需要说明的是, 本文采用的降水预报资料是 $24 \mathrm{~h}$ 间隔的时段累积量分级预报,将未来 $24 \mathrm{~h}$ 降雨预 报量平均分配到 $24 \mathrm{~h}$ 作为模型输人. 由于预报资 料的局限性,这种时间上的均化处理方式(没有考 虑雨型分布)不可避免地带来径流模拟过程坦化 现象.

水库在不进行下泄条件下 $24 \mathrm{~h}$ 的小雨、中雨、

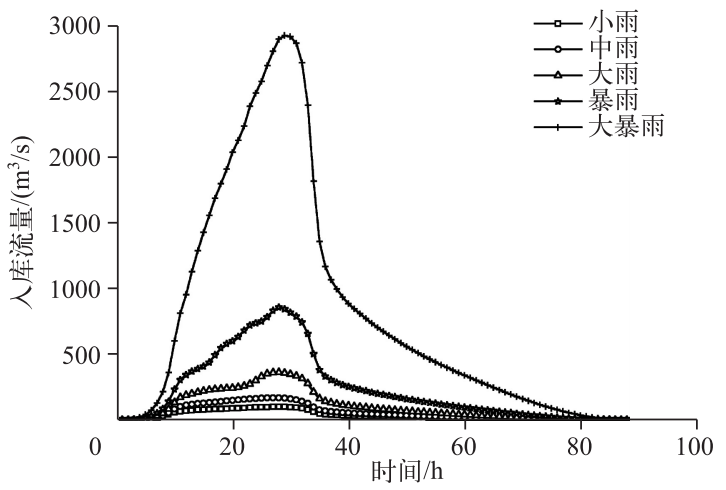

图 4 各量级降雨条件下响洪甸人库流量过程线

Fig.4 The flow hydrograph of flood discharge of Xianghongdian Reservoir under the condition of rainfall 大雨的最大人库流量差别不明显, 分别为 $100 、 170 、 364 \mathrm{~m}^{3} / \mathrm{s}$; 暴雨条件下最大人库流量增大至 $859 \mathrm{~m}^{3} / \mathrm{s}$; 大 暴雨条件下迅速增大至 $2930 \mathrm{~m}^{3} / \mathrm{s}$. 在小雨、中雨和大雨预报条件下水库水位峰值分别为 $125.17 、 125.29$ 、 $125.59 \mathrm{~m}$, 涨幅较小; 暴雨条件下水库水位峰值为 $126.27 \mathrm{~m}$, 尤其是大暴雨条件下显著上涨至 $129.01 \mathrm{~m}$, 涨幅 达 $4.01 \mathrm{~m}$.

以 2016 年 6 月 12 日 2 时水位 ( $125 \mathrm{~m}$, 汛限水位) 为起点, 根据人库洪量及水库参数并参考下游允许泄 量对不同历时库水位上涨 $0 、 0.5 、 1.0 、 2.0 \mathrm{~m}$ 的下泄方案流量进行计算, 不同雨量级和下泄时间对于不同目标 库水位对应着不同的泄流量 (表 3 )。

表 3 不同历时下泄方案流量 $\left(\mathrm{m}^{3} / \mathrm{s}\right)$

Tab.3 Discharges scheme of specified water level rise for different duration

\begin{tabular}{|c|c|c|c|c|c|c|c|c|}
\hline \multirow{2}{*}{ 预见期 } & \multicolumn{4}{|c|}{ 暴雨量级 } & \multicolumn{4}{|c|}{ 大暴雨量级 } \\
\hline & $0 \mathrm{~m}$ & $0.5 \mathrm{~m}$ & $1.0 \mathrm{~m}$ & $2.0 \mathrm{~m}$ & $0 \mathrm{~m}$ & $0.5 \mathrm{~m}$ & $1.0 \mathrm{~m}$ & $2.0 \mathrm{~m}$ \\
\hline $72 \mathrm{~h}$ & 298.30 & 181.60 & 63.93 & - & 1014.78 & 898.07 & 780.40 & 541.40 \\
\hline $48 \mathrm{~h}$ & 447.45 & 272.40 & 95.89 & - & 1522.16 & 1347.11 & 1170.60 & 812.09 \\
\hline $24 \mathrm{~h}$ & 894.91 & 544.79 & 191.78 & - & 3044.33 & 2694.21 & 2341.20 & 1624.19 \\
\hline $12 \mathrm{~h}$ & 1789.81 & 1089.58 & 383.56 & - & 6088.66 & 5388.43 & 4682.41 & 3248.38 \\
\hline
\end{tabular}

小雨、中雨、大雨量级降水过程对水库水位影响较小, 根据降水预报可适当预泄或在降雨汇人水库进行 实时调度, 对于暴雨及以上量级的降水预报应提前做好准备, 根据预报降雨量及径流深, 推求人库流量过程 线,综合人库洪量和调度目标对水库做合理预泄. 
在暴雨量级下,以库水位涨幅为零为调度目标, $12 、 24 、 48 、 72 \mathrm{~h}$ 对应下泄流量分别为 1789.81 、894.91、 $447.45 、 298.30 \mathrm{~m}^{3} / \mathrm{s}$, 由于人库流量过程线中流量峰值为 $859 \mathrm{~m}^{3} / \mathrm{s}$, 考虑到消峰作用只有泄流历时大于 $48 \mathrm{~h}$ 的下泄方案为合理. 同理可对涨幅 $0.5 、 1.0 \mathrm{~m}$ 的下泄方案进行分析.

暴雨量级降雨条件下, 在下泄为零情况下水库最高水位为 $126.27 \mathrm{~m}$, 达不到 $2.0 \mathrm{~m}$ 涨幅, 对降雨汇流过 程结束时相对起始状态水位涨幅 $2.0 \mathrm{~m}$ 的调度目标无需对库容预泄. 在大暴雨量级降水条件下, 人库流量峰 值为 $2930 \mathrm{~m}^{3} / \mathrm{s}$, 若以降雨结束时保持起始状态水位 $(0 \mathrm{~m})$ 为调度目标, $72 \sim 48 \mathrm{~h}$ 内调度方案都能起到消峰作 用. 对水文涨幅 $0.5 、 1.0 、 2.0 \mathrm{~m}$ 不同调度目标, 选取不同的调度方案起到消峰错峰和减缓下游压力的作用.

除防洪以外供水发电灌溉也是水库的重要功能, 掌握降水预报的偏差和不确定性, 可以避免大量弃水 损失. 小雨、中雨的降雨量期望值均大于小雨、中雨雨量值范围 (表 2), 在此预报条件下由于小雨、中雨、大 雨对水库水位影响较小, 在此可不做分析. 以未来 $24 \mathrm{~h}$ 预报为暴雨、大暴雨, 用各雨量级降雨量期望值上限 对水库调度预泄量计算来作为水库的预泄值, 并结合实时雨水情进行相应调整, 可以对水库库容做到有效 控制, 既满足了水库防洪要求, 保证水库及下游安全同时也保障了水库的生产效益.

\section{2 不同预见期降水预报应用策略}

利用降水预报和实况结果分别计算不同量级 $N A 、 N B 、 N C 、 N D$ 值得到 $R O C$ 曲线 (图 5), 以进一步检验不 同预见期的不同量级降水预报性能. 各时次各量级的降水预报 $R O C$ 特征曲线都在对角线以上,表明降水预 报对各量级的降水都有正预报技巧. 但是各量级的 $A U C$ 面积随预见期增长呈显著下降, 且超过 $24 \mathrm{~h}$ 预见期 的降水预报下降趋势明显.

降雨预报性能随预见期缩短有所提高, 应根据最新降水预报对水库调度方案进行调整, 制定更合理的 预泄或蓄水方案. 不同预见期的降雨预报体现在对某一时段降雨量预报的时间长度,对于同一地区前期降 雨条件相同情况下, 降雨径流关系是确定的, 推求的人库流量过程线以及对应的人库洪量一定. 对未来 $24 \mathrm{~h}$ 预报为暴雨, 响洪甸水库在预见期为 $72 、 48 、 24 \mathrm{~h}$ 所设计的下泄流量分别为 $295.5 、 443.3 、 886.6 \mathrm{~m}^{3} / \mathrm{s}$. 降水预 报的预见期越长, 对水库预泄的时间就可以较长, 相应下泄流量变小. 降低预泄流量的意义就在于避免短时 下泄大水量, 降低对下游的压力做到消峰错峰效果, 也可避免降水预报出现较大误差造成后期无水可蓄; 若 后期降雨增大可适时加大下泄量增加机动性能.

\section{3 转折性天气降水预报应用策略}

转折性天气是指从无降雨到有降雨或者从有降雨到无降雨的天气变化过程. 转折性天气的降水范围和 强度预报难度要高于稳定型天气, 但是转折性天气降水预报对于水库调度策略制定非常重要. 对所有转折 性天气个例分不同预见期分析其 CTS 评分、空报率、漏报率(图 6).

$96 \mathrm{~h}$ 以上预见期预报 CTS 评分低于或者接近于 $20 \%$, 空报率和漏报率均高于 $60 \% ; 72 \mathrm{~h}$ 以内空报率和 漏报率明显下降, 尤其是 $24 \mathrm{~h}$ 预见期空报率和漏报率均在 50\% 以下, CTS 评分也提高到了 $38.2 \%$, 短预见期 的转折性天气降水预报仍然有指示意义(图 6).

以 2016 年 6 月 20 日至 10 月 11 日响洪甸水库运行状态为例,进一步分析转折性天气降水预报在水库 调度中的应用. 图 7 为 6 月 20 日至 10 月 11 日响洪甸水库流域的面雨量、水库的下泄流量和水库水位过 程线.

6 月 30 日前后大别山库区出现了一次强降雨过程, 由于水库未进行预泄导致水库水位快速上升, 峰值 达到 $129.41 \mathrm{~m}$, 随即水库加大下泄流量, 最大泄流量达 $1000 \mathrm{~m}^{3} / \mathrm{s}$, 使水位快速回落到起始水平, 未进行蓄水. 在此次降雨过程结束后至 10 月 11 日期间降雨较少且雨量级较小, 最大日面雨量仅 $35 \mathrm{~mm}$, 其中 7 月 22 日 至 8 月 1 日、 8 月 9 日至 9 月 14 日、 9 月 17 日至 9 月 26 日无降雨, 10 月 11 日水位落至 $116.95 \mathrm{~m}$. 至 9 月 17 日期间水库泄流量基本保持在 $123 \mathrm{~m}^{3} / \mathrm{s}$ 左右, 主要用来发电, 9 月 17 日开始水库发电洞关闭. 由于没有做 到对转折性天气下水库的合理调度, 未对 6 月 30 日的强降水做到合理预泄和水资源有效利用, 致使水库最 高水位达 $129.41 \mathrm{~m}$, 超汛限水位 $4.41 \mathrm{~m}$, 超正常蓄水位 $1.41 \mathrm{~m}$; 因下泄造成汛限水位和正常蓄水位之间的库 容没有有效利用, 后期又因降水少、发电消耗导致库水位逐渐降低, 9 月 17 日起关闭发电洞, 影响了生产 效益.

由此可见, 转折性天气预报对于水库的调度非常重要, 从无降雨到有降雨的转折过程需要对降雨预报 

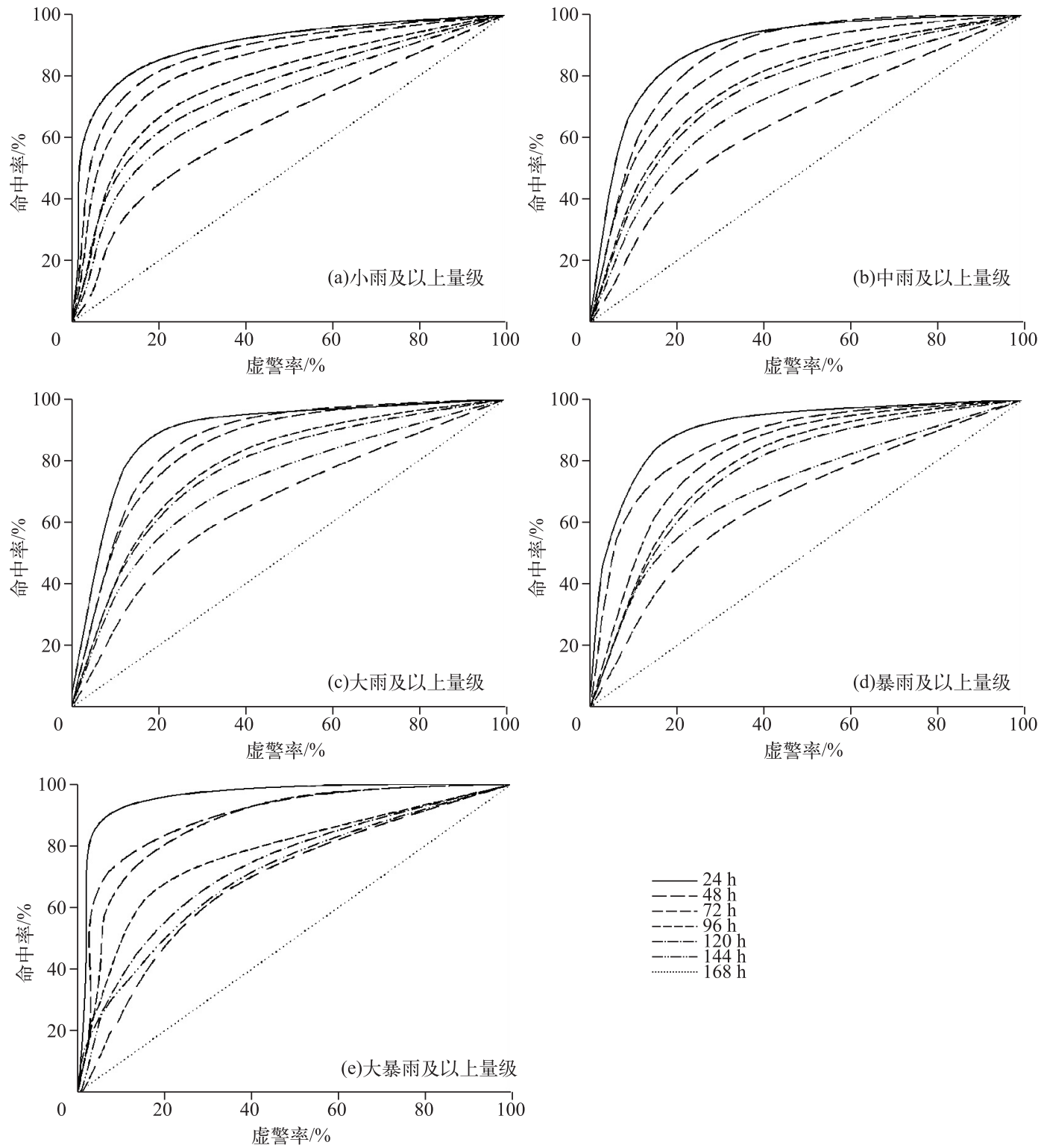

图 5 小雨 $(\mathrm{a})$ 、中雨 $(\mathrm{b}) 、 大$ 雨 $(\mathrm{c})$ 、暴雨 $(\mathrm{d})$ 、大暴雨 $(\mathrm{e})$ 及其以上量级降水预报 $R O C$ 曲线

Fig.5 The ROC curves of different precipitation levels

量级、预见期、水库参数及调度的目标综合考虑进行水库调度, 起到消峰错峰的作用确保水库安全; 从有降 雨到无降雨的过程则需要考虑前期降雨量、水库当前状态以及雨后水库供水、发电、灌溉等方面用水的需 求,对水库调度方案进行调整,做到汛前弃水保安全,汛后蓄水保效益.

\section{5 结论}

1) 大别山库区各量级的降水预报都有正预报技巧, 但随预见期延长显著下降. $72 \mathrm{~h}$ 预见期降雨预报具 有较高的预报性能; $96 \mathrm{~h}$ 及以上预见期降水预报性能明显下降,特别是对大暴雨及其以上量级的降水预报 性能显著下降. 大别山库区降水预报量级上呈现出过度预报的现象, 但降水过程预报对水库调度仍有较好 


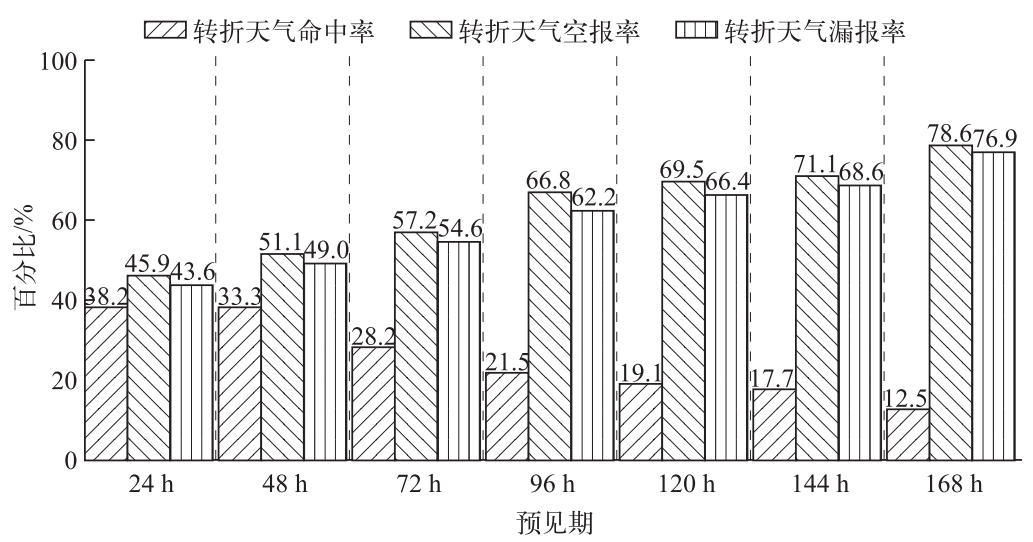

图 6 转折性天气 CTS 、空报率、漏报率随预见期变化

Fig. 6 The variation of CTS score, false alarm rate and missing alarm rate of intransition weather with different lead time

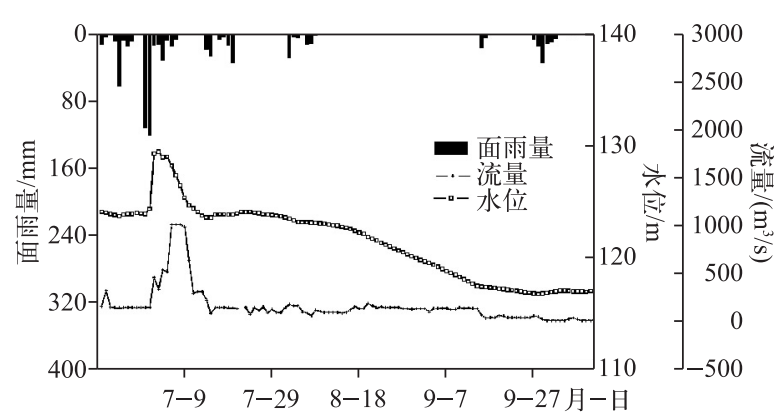

图 7 响洪甸水库面雨量、泄流量、水位过程线

Fig.7 Areal main rainfall, discharge and water level of Xianghongdian Reservoir
的应用价值, 可综合人库洪量和调度目标对水 库做合理预泄, 并结合实时雨水情进行相应 调整.

2) 转折性天气预报一直是天气预报的难 点. 虽大别山库区转折性天气预报性能与连 续性降水预报性能相比明显较低, 但可从长预 见期的降水预报获取未来降水过程及其可能 发生转折的信息,初步制定转折性天气下的水 库调度方案, 随着天气系统的演变发展, 再根 据短预见期的降水预报进行调度方案的调整.

3 ) 本文仅分析了气象台公开发布的降水 预报准确率及其在单一水库调度方面的应用 对策, 未涉及降水预报误差原因及改进方法研

究. 分析不同预见期、不同降雨强度对预报准确率的影响,并提出其订正方法是值得进一步研究的问题; 同 时, 利用较长预见期的降水预报进行多水库联合调度研究, 可以提高水库水资源利用率并有效减轻下游洪 水灾害.

\section{6 参考文献}

[ 1 ] Liu Ning. Flood control \& drought resistance and risk management of flood and drought. China Flood \& Drought Management , 2012, 22(2) : 1-4. [刘宁. 防汛抗旱与水旱灾害风险管理. 中国防汛抗旱, 2012, 22(2) : 1-4.]

[ 2 ] Zhai Panmao, Li Lei, Zhou Baiquan et al. Progress on mechanism and prediction methods for persistent extreme precipitation in the Yangtze-Huai River Valley. Journal of Applied Meteorological Science, 2016, 27(5) : 631-640. [翟盘茂, 李 蕾, 周佰铨等. 江淮流域持续性极端降水及预报方法研究进展. 应用气象学报, 2016, 27(5): 631-640.]

[ 3 ] Huo Shiqing, Wang Chunqing, Xu Zhuoshou. Progress on hydro-meteorological information and forecasting of the Yellow River in recent ten years. Yellow River, 2016, 38(10) : 36-39. [霍世青, 王春青, 许卓首. 近 10 a 黄河水文气象情报 预报工作进展. 人民黄河, 2016, 38(10): 36-39.]

[ 4 ] Zhang Hexi, Chi Daocai, Wang Yongtao et al. Applied research of the rainfall forecast model based on NNBR and MonteCarlo algorithm. Research of Soil and Water Conservation, 2014, 21(2): 106-110. [张和喜, 迟道才, 王永涛等. 基于 NNBR 与蒙特卡洛算法的降雨量预报模型应用研究. 水土保持研究, 2014, 21(2)：106-110.] 
[ 5 ] Zhang Qianyu, Xu Youpeng, Lei Chaogui et al. Risk assessment of flood based on dynamic simulation in downstream of reservoirs in coastal area of southeast China. J Lake Sci, 2016, 28(4) : 868-874. DOI:10. 18307/2016. 0421. [张倩玉, 许有鹏，雷超桂等. 东南沿海水库下游地区基于动态模拟的洪涝风险评估. 湖泊科学, 2016, 28(4) : 868-874.]

[ 6 ] Cui Chunguang, Peng Tao, Shen Tieyuan et al. The flood forecast test on QPF coupling with hydrological model in flood season in medium and small catchment. Meteorological Monthly, 2010, 36(12): 56-61. [崔春光, 彭涛, 沈铁元等. 定 量降水预报与水文模型耦合的中小流域汛期洪水预报试验. 气象, 2010, 36(12): 56-61.]

[ 7 ] Van Steenbergen N, Willems P. Rainfall uncertainty in flood forecasting: Belgian case study of Rivierbeek. Journal of Hydrologic Engineering, 2014, 19(19) : 554-555.

[ 8 ] Lin CA, Wen L, Lu G et al. Real-time forecast of the 2005 and 2007 summer severe floods in the Huaihe River Basin of China. Journal of Hydrology, 2010, 381 ( 1 ) : 33-41.

[ 9 ] Diakakis M. Rainfall thresholds for flood triggering. The case of Marathonas in Greece. Natural Hazards, 2011, 60(3) : 789-800.

[10] Huang Xiaoxiang, Yao Cheng, Li Zhijia et al. Application of grid-Xinanjiang model in the upstream of Yuqiao Basin, Tianjin. J Lake Sci, 2016, 28(5) : 1134-1140. DOI: 10.18307/2016.0524. [黄小祥, 姚成, 李致家等. 栅格新安江模 型在天津于桥水库流域上游的应用. 湖泊科学, 2016, 28(5): 1134-1140.]

[11] Zhang Xingxing, Zhu Chengli, Peng Shizhang et al. Accuracy analysis of rainfall forecast and its application to improvement of utilization rate of rainfall. Journal of Hohai University: Natural Sciences, 2014, 42(3): 230-233. [张星星, 朱成 立, 彭世彰等. 降水预报准确度分析及其在提高降水利用率中的作用. 河海大学学报: 自然科学版, 2014,42 (3) : 230-233.]

[12] Shi Zhigang. Study on the characteristics of soil and water loss and integrated control in the Dabieshan Area in the west of Anhui Province. Research of Soil and Water Conservation, 2012, 19(5):39-42. [史志刚. 皖西大别山区水土流失特征 及其综合防治研究. 水土保持研究, 2012, 19(5) : 39-42.]

[13] Ye Jinyin, Li Zhijia, Liu Jing et al. Identification of early meteorological risk warning indicators for flash flood disasters. Torrential Rain and Disasters, 2016, 35(1) : 25-30. [叶金印, 李致家, 刘静等. 山洪灾害气象风险预警指标确定方 法研究. 暴雨灾害, 2016, 35(1) : 25-30.]

[14] Liu Yihua, Lu Yanrong, Zhou Qiang et al. Application of HBV model to the study on risk precipitation in different grades in Batang River region. Research of Soil and Water Conservation, 2015, 22(2): 224-228. [刘义花, 鲁延荣, 周强等. HBV 水文模型在玉树巴塘河流域洪水临界雨量阈值研究中的应用. 水土保持研究, 2015, 22(2) : 224-228.]

[15] Ye Jinyin, Gu Weiqi, Li Qiaoling et al. Application of ECMWF ensemble forecasts in Jiangjiaji Catchment of Huaihe River Basin. Journal of Hohai University: Natural Sciences, 2016, 44(6): 471-476. [叶金印, 顾玮琪, 李巧玲等. ECMWF 集合预报在淮河蒋家集流域的应用. 河海大学学报：自然科学版, 2016, 44(6)：471-476.]

[16] Ye Jinyin, Shao Yuehong, Li Zhijia. Flood forecasting based on TIGGE precipitation ensemble forecast. Advances in Meteorology, 2016. DOI:10. 1155/2016/9129734.

[17] Zhang Bing, Wei Jiansu, Wang Wenlan et al. A method for verifying precipitation prediction of transition weather. Meteorological Science and Technology, 2012, 40(3): 411-416. [张冰, 魏建苏, 王文兰等. 转折性天气降水预报检验方法 及应用. 气象科技, 2012, 40(3): 411-416.]

[18] Liu Lin, Chen Jing, Cheng Long et al. Study of the ensemble-based forecast of extremely heavy rainfalls in China. Acta Meteorologica Sinica, 2013, 71(5)：853-866. [刘琳, 陈静, 程龙等. 基于集合预报的中国极端强降水预报方法研究. 气象学报, 2013, 71(5): 853-866.]

[19] Huaihe River Water Resources Committee. Practical hydrological forecast schemes of drainage in Huaihe River Basin. Jinan: Huanghe Press, 2002: 125-152. [淮河水利委员会. 淮河流域淮河水系实用水文预报方案. 济南: 黄河出版 社, 2002: 125-152.] 